\title{
Computer-related health problems among university students in Majmaah region, Saudi Arabia.
}

\author{
Mohamed Sherif Sirajudeen ${ }^{1 *}$, Hariraja Muthusamy ${ }^{1}$, Mazen Alqahtani ${ }^{1}$, Mohamed Waly ${ }^{2,3}$, Abdul \\ Khadar Jilani ${ }^{4}$ \\ ${ }^{1}$ Department of Physical Therapy and Health Rehabilitation, College of Applied Medical Sciences, Majmaah \\ University, Majmaah 11952, Saudi Arabia \\ ${ }^{2}$ Department of Medical Equipment Technology, College of Applied Medical Sciences, Majmaah University, Majmaah \\ 11952, Saudi Arabia \\ ${ }^{3}$ Higher Institute for Engineering, El Shorouk Academy, Cairo, Egypt \\ ${ }^{4}$ Computer Science and Information Technology College, Majmaah University, Majmaah 11952, Saudi Arabia
}

\begin{abstract}
Introduction: The increased reliance on computer results in crucial health issues among the users. This research aimed to study the prevalence and factors associated with Computer-related health problems among University students in Majmaah region, Saudi Arabia.

Materials and methods: 146 students were selected for this cross-sectional study using conveniencesampling technique. Data regarding personal characteristics, computer usage and prevalence of Musculoskeletal disorders (MSDs), Visual symptoms and sleep disorders were collected by a valid, reliable and self-administered questionnaire.

Results: The prevalence of MSDs (any one body region), Visual symptoms (any one symptom) and sleep disorders was $52.7 \%, 54.8 \%$ and $56.8 \%$ respectively. Female gender, Laptop use without external mouse and inadequate breaks were associated with MSDs $(\mathbf{P}<\mathbf{0 . 0 5})$. Extensive smart phone use was associated with sleep disorders $(\mathbf{P}<\mathbf{0 . 0 5})$.

Conclusion: The measures to promote the awareness about health and safety issues related to computer use among the university students should be given utmost priority. Moreover, the culture of reporting injuries and relevant issues should be encouraged among the student community to enhance early detection and intervention.
\end{abstract}

Keywords: Computer-related health problems, Computer use, University students, Ergonomics.

Accepted on March 22, 2018

\section{Introduction}

Computers play an essential role in human life. Computers had revolutionized educational, communication, transport, commerce, healthcare and entertainment sectors. This increased reliance on computer results in crucial health issues among the users [1]. There is steady increase in the computer penetration among residents of Saudi Arabia in the past three years from $43 \%$ in 2007 to $53 \%$ in 2009 [2].

"Ergonomics is the scientific study of human work" [3]. The goal of Ergonomics is to design the job to fit the worker thereby enhancing working efficiency, comfort, health and safety [4]. Neglecting the principles of ergonomics during computer utility raises the risk of health problems [5]. Musculoskeletal disorders (MSDs), Visual symptoms and Insomnia are the common health problems amongst computer users [6-9].
"Musculoskeletal disorders" covers an extensive range of conditions affecting the musculoskeletal system including the joints, muscles, tendons, ligaments, peripheral nerves, and supporting blood vessels $[10,11]$. MSDs are inflammatory and degenerative in nature with symptoms like pain, numbness, tingling, aching, stiffness, or burning [12]. Improper workstation design and faulty posture are risks associated with computer use. Working in static sitting positions for prolonged duration, results in reduced circulation, joint pain and stiffness. Extended period of continuous work raises the risk of MSDs, and leads to prolonged disability [13].

Daily $3 \mathrm{~h}$ usage of computer also leads to a risk of developing visual complaints. Common visual symptoms include eyestrain, headache and blurred vision. Prevalence of visual complaints among computer users ranges from $64 \%$ to $90 \%$. Globally almost 60 million computer users suffer from visual complaints and a million new cases of visual complaints arise 
each year [14]. In a study of association between daily Computer work duration and sleep disturbances, Labbafinejad et al. observed that that subjects who spent longer durations each day on computer work tended to experience sleep disturbances [15].

Not many studies that focused on health issues related to the use of computers among college going students; still exposure to risk may be identical to that of employees who use computers. Noack-Cooper et al. compared the pattern of computer use between the college students and the other group that includes computer professionals. Younger graduate students in their study reported average weekly computer use of $33.7 \mathrm{~h}$, similar to that reported by younger professionals [16]. In a study by Lorusso et al. among Italian college students using desktop computers to determine the prevalence of musculoskeletal symptoms observed that $69 \%, 53 \%, 49 \%$ of the participants experienced neck, hand/wrist and shoulder symptoms respectively [17].

Logaraj et al. performed a cross sectional survey to study the occurrence of visual complaints amongst engineering students in India using computers. The results showed that, the prevalence of visual complaints was found to be $81.9 \%$ among engineering students [14]. University students deserves attention and studies addressing this population is very important to study the pattern of various health problems, associated factors and measures to prevent them. Hence, this study first of its kind in Saudi Arabia was aimed to determine the prevalence and factors associated with health problems among computer science and information technology students of Majmaah University, Saudi Arabia.

\section{Materials and Methods}

146 University students in Majmaah region were selected by convenience sampling method to participate in this crosssectional study. Subjects with pregnancy, chronic systemic illness, recent fractures or surgeries were excluded from the study. The researchers obtained the informed consent from the all study participants. The ethical approval was obtained from Ethics committee of Majmaah University. The data collected were handled confidentially.

The data related to personal characteristics, computer usage, and the prevalence of musculoskeletal disorders, visual symptoms and sleep disorders were collected using a valid, reliable and pretested questionnaire. The items related to age, gender, height, weight, Body Mass Index (BMI) and department of study were included in Personal characteristics section. The BMI was classified in accordance with the international classification system of the WHO [18]. The computer usage section consisted of items like years of computer use, daily usage of desktop, laptop, smart phone and other gadgets in hours, breaks and source of ergonomic tips. Musculoskeletal disorders section consists of a series of items adapted from Nordic Musculoskeletal Questionnaire (NMQ) [19]. The NMQ is a predominantly used valid and reliable tool in musculoskeletal surveillance studies. The case definition used in this study was broad enough to differentiate MSDs that were significant to create problems at work from minor complaints.

Musculoskeletal disorder is defined as having had a pertinent symptom such as pain, numbness, tingling, aching, stiffness, or burning sensation that has lasted for a week or more and or occurred monthly with at least moderate pain on average over the past one year. The level of pain was determined with a 5point pain scale [20]: "none/no pain," "mild/minimal," "moderate," "severe," and "worst pain ever in my life." The scientists at the National Institute for Occupational Safety and Health developed and standardized this definition of a musculoskeletal disorder [21].

An ailment of MSD was ascertained by asking a question "Had you presented with any kind of discomfort or pain on the musculoskeletal system over the past one year that is related to the use of computers?" MSD duration was evaluated by the question "How long does the pain or discomfort usually last ( $24 \mathrm{~h}$ or less, $24 \mathrm{~h}$ to 1 week, $>1$ week to 1 month, $>1$ month to 6 months, or $>6$ months)?" MSD frequency was evaluated by the question "How many times have you had the pain or discomfort (once every 6 months or less, once every 2-3 months, once a month, once a week, or more often than once a week)?" Participants need to provide appropriate responses for questions related to affected body region if any.

The study participants were asked to report any visual symptoms (headache, burning eyes, redness, and double vision, focusing problem and eye fatigue) experienced while using computer at either university or home within the past 12 months. They were requested to tick whether they had experienced the symptoms, almost never ( 2 times in a year or less), rarely (once every 2-3 months), sometimes (once in a month), regularly (once in a week) or almost always (daily) during or after computer use. Each symptom was dichotomized into "yes" (sometimes, frequently or almost always) and "no" (almost never or rarely) responses.

The sleep disorders (Insomnia) were screened using Athens Insomnia Scale (AIS). The AIS is a valid and reliable tool to screen sleep disturbances and to determine clinically substantial insomnia. Out of eight AIS items, the first five addresses participant's night-time symptoms (difficulty in sleep initiation, difficulty in maintaining sleep and early morning awakening) and last three items addresses the daytime impact due of sleep disturbances (wellbeing, functioning capacity and daytime sleepiness). The subjects should respond to the items if they faced any sleep disturbances at least weekly thrice during the preceding month. The total score ranged from 0 to 24. The score " $\geq 6$ " is considered as presence of insomnia $[22,23]$.

\section{Statistical analysis}

The data were analysed using SPSS for Windows. Descriptive statistics was performed for personal characteristics and computer usage. The prevalence of MSDs (for each body region) was determined by considering the number of 
participants affected in that body region and dividing it by the total number of participants who answered the questionnaire. The prevalence of visual symptoms calculated, by including the student population with the existing symptoms and dividing it by total number of students who answered the questionnaire. The prevalence of insomnia was considered by taking the number of cases of insomnia and dividing it by the total number of students who answered the questionnaire. The association between selected factors and prevalence of MSDs, visual symptoms and insomnia were analysed using the chisquare test of association. $5 \%$ level of probability was used to indicate statistical significance.

\section{Results}

The data regarding personal characteristics of the participants is presented in Table 1. Majority of participants were female (57.5\%) when compared to males $(42.5 \%)$. Nearly $54.8 \%$ of participants belonged to 22-24 age group. With regard to body mass index, majority of participants (61\%) were normal weight whereas $16.4 \%$ and $8.9 \%$ of them were underweight and obese respectively. Among the participants, 56.2\% were Information technology students and $43.8 \%$ were computer science students.

Table 1. Personal characteristics of participants.

\begin{tabular}{|c|c|c|c|}
\hline Parameter & Characteristics & Frequency & Percent \\
\hline \multirow{2}{*}{ Gender } & Male & 62 & $42.5 \%$ \\
\hline & Female & 84 & $57.5 \%$ \\
\hline \multirow{4}{*}{ Age } & From $18-20$ & 8 & $5.5 \%$ \\
\hline & From 20-22 & 55 & $37.7 \%$ \\
\hline & From 22-24 & 80 & $54.8 \%$ \\
\hline & More than 24 & 3 & $2.1 \%$ \\
\hline \multirow{4}{*}{ BMI } & Under weight & 24 & $16.4 \%$ \\
\hline & Normal weight & 89 & $61 \%$ \\
\hline & Over weight & 20 & $13.7 \%$ \\
\hline & Obese & 13 & $8.9 \%$ \\
\hline \multirow{2}{*}{ Department } & Information technology & 82 & $56.2 \%$ \\
\hline & Computer science & 64 & $43.8 \%$ \\
\hline
\end{tabular}

The data related to desktop usage is presented in Table 2. Majority of the participants $(45.9 \%)$ reported $5-10$ y of computer use. $87 \%$ of the participants were using desktop less than $3 \mathrm{~h}$ per day. $87 \%$ and $87.7 \%$ of the participants reported less than $3 \mathrm{~h}$ of keyboard and mouse per day respectively. Majority of participants $(51.4 \%)$ took break once in $2 \mathrm{~h}$ during computer use. With regard to the source of ergonomic knowledge, $14.4 \%$ of the participants were not aware of ergonomics and $63.7 \%, 51.4 \%$ and $50.7 \%$ of the participants mentioned internet, family and friends and college as their source of ergonomic knowledge respectively.

Table 2. Computer usage of participants.

\begin{tabular}{|c|c|c|c|}
\hline Parameter & Characteristics & Frequency & Percent \\
\hline \multirow{3}{*}{$\begin{array}{l}\text { Computer } \\
\text { (y) }\end{array}$} & Less than $5 \mathrm{y}$ & 27 & $18.5 \%$ \\
\hline & From 5-10 y & 67 & $45.9 \%$ \\
\hline & From $10-15$ y & 52 & $35.6 \%$ \\
\hline \multirow{3}{*}{$\begin{array}{l}\text { Daily desktop } \\
\text { use }(\mathrm{h})\end{array}$} & Less than $3 \mathrm{~h}$ & 127 & $87 \%$ \\
\hline & From 3-6 h & 14 & $9.6 \%$ \\
\hline & More than $6 \mathrm{~h}$ & 5 & $3.4 \%$ \\
\hline \multirow{3}{*}{$\begin{array}{l}\text { Daily key board } \\
\text { Use }(h)\end{array}$} & Less than $3 \mathrm{~h}$ & 127 & $87 \%$ \\
\hline & From 3-6 h & 14 & $9.6 \%$ \\
\hline & More than $6 \mathrm{~h}$ & 5 & $3.4 \%$ \\
\hline \multirow{3}{*}{$\begin{array}{l}\text { Daily mouse use } \\
\text { (h) }\end{array}$} & Less than $3 \mathrm{~h}$ & 128 & $87.7 \%$ \\
\hline & From $3-6 \mathrm{~h}$ & 16 & $11 \%$ \\
\hline & More than $6 \mathrm{~h}$ & 2 & $1.4 \%$ \\
\hline \multirow{3}{*}{ Take breaks } & Once in $2 \mathrm{~h}$ & 75 & $51.4 \%$ \\
\hline & Once in $2-4 \mathrm{~h}$ & 57 & $39 \%$ \\
\hline & Once in $4 \mathrm{~h}$ or more & 14 & $9.6 \%$ \\
\hline \multirow{7}{*}{$\begin{array}{l}\text { Source } \\
\text { ergonomics } \\
\text { knowledge }\end{array}$} & Not aware & 21 & $14.4 \%$ \\
\hline & College & 74 & $50.7 \%$ \\
\hline & Family and friends & 75 & $51.4 \%$ \\
\hline & Internet & 93 & $63.7 \%$ \\
\hline & News paper & 11 & $7.5 \%$ \\
\hline & Television & 36 & $24.7 \%$ \\
\hline & Others & 26 & $17.8 \%$ \\
\hline
\end{tabular}

The details related to daily usage of laptop, smart phones and other gadgets by the participants are summarized in Table 3. Majority of the participants $(43.8 \%)$ reported 3-6 h of laptop usage. $59.6 \%$ of participants performed less than $3 \mathrm{~h}$ of keying in laptop. When using laptop, only $26.7 \%$ and $37 \%$ of participants reported using external keyboard and mouse respectively. Majority of the participants (87.7\%) reported using I pad or any other gadget less than $3 \mathrm{~h}$.

Table 3. Laptop, smart phones and other gadget usage of participants.

\begin{tabular}{llll}
\hline Parameter & Characteristics & Frequency & Percent \\
\hline \multirow{2}{*}{ Daily laptop use $(\mathrm{h})$} & Less than 3 $\mathrm{h}$ & 44 & $30.1 \%$ \\
\cline { 2 - 4 } & From 3-6 h & 64 & $43.8 \%$ \\
\cline { 2 - 4 } & More than 6 h & 38 & $26 \%$ \\
\hline \multirow{2}{*}{$\begin{array}{l}\text { Daily key board use } \\
\text { (h) }\end{array}$} & Less than 3 h & 87 & $59.6 \%$ \\
\cline { 2 - 4 } & From 3-6 h & 45 & $30.8 \%$ \\
\hline
\end{tabular}




\begin{tabular}{|c|c|c|c|}
\hline & & & \\
\hline & More than $6 \mathrm{~h}$ & 14 & $9.6 \%$ \\
\hline \multirow{2}{*}{$\begin{array}{l}\text { Use external key } \\
\text { board }\end{array}$} & Yes & 39 & $26.7 \%$ \\
\hline & No & 107 & $73.3 \%$ \\
\hline \multirow{2}{*}{ Use external mouse } & Yes & 54 & $37 \%$ \\
\hline & No & 92 & $63 \%$ \\
\hline \multirow{3}{*}{$\begin{array}{l}\text { Daily smart phone } \\
\text { use }(h)\end{array}$} & Less than $3 \mathrm{~h}$ & 15 & $10.3 \%$ \\
\hline & From 3-6 h & 47 & $32.2 \%$ \\
\hline & More than $6 \mathrm{~h}$ & 84 & $57.5 \%$ \\
\hline \multirow{3}{*}{$\begin{array}{l}\text { Daily I pad or } \\
\text { another gadget use } \\
\text { (h) }\end{array}$} & Less than $3 \mathrm{~h}$ & 128 & $87.7 \%$ \\
\hline & From 3-6 h & 15 & $10.3 \%$ \\
\hline & More than $6 \mathrm{~h}$ & 3 & $2.1 \%$ \\
\hline
\end{tabular}

The prevalence of MSDs in any one body region among students in this study was $52.7 \%$ (Figure 1). The pattern of MSDs among the participants showed that highest prevalence was neck disorders (45.9\%) followed by upper back $(29.4 \%)$, lower back $(26.7 \%)$, shoulders $(21.2 \%)$, wrists/ hand $(20.5 \%)$, knee $(20.5 \%)$, ankles/feet (14.4\%), elbow (11\%), and hip/thigh $(8.9 \%)$.
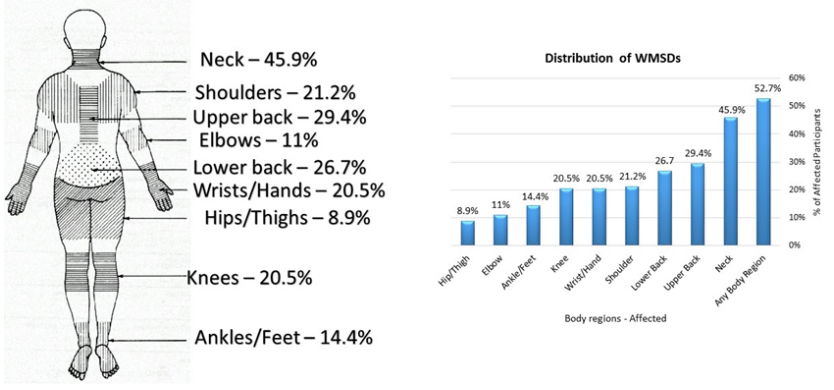

Figure 1. Prevalence and distribution of MSDs.

The prevalence of visual symptoms and insomnia are presented in Table 4. About $22.6 \%$ of participants reported wearing corrective lens. The prevalence of any one visual symptom among the participants was $54.8 \%$. The pattern of visual symptoms among the participants showed that highest prevalence was headache $(45.2 \%)$ followed by eye fatigue (35.6\%), focusing problem (34.9\%), burning sensations $(23.3 \%)$, redness $(23.3 \%)$ and double vision $(23.3 \%)$. The prevalence of sleep disorders among the participants was $56.8 \%$.

The association between personal characteristics and prevalence of MSDs are summarized in Table 5. Female students were more likely to develop MSDs of neck $(p=0.03)$, upper back $(p=0.001)$, lower back $(p=0.004)$ and ankle/feet $(p=0.01)$. The MSDs of upper back was associated with age $(p=0.016)$ and occurred most commonly in 22-24 age group. Over weight students were more likely to develop MSDs of knee $(p=0.02)$. The association between desktop usage and the prevalence of MSDs are presented in Table 6. The years of computer usage was associated with prevalence of MSDs of upper back $(p=0.002)$, hip/thigh $(p=0.03)$, knees $(p=0.001)$ and ankle/feet $(\mathrm{p}=0.006)$ and occurred most commonly among the participants reported using computer less than $5 \mathrm{y}$. The participants who reported availing less frequent breaks (once in $4 \mathrm{~h}$ or more) during computer use were more likely to develop MSDs of shoulder $(\mathrm{p}=0.005)$, elbow $(\mathrm{p}=0.02)$, and wrist $/$ hands $(\mathrm{p}=0.001)$.

Table 4. Prevalence of visual complaints and insomnia among participants.

\begin{tabular}{|c|c|c|c|}
\hline Parameter & Characteristics & Frequency & Percent \\
\hline \multirow{2}{*}{ Corrective lens } & Yes & 33 & $22.6 \%$ \\
\hline & No & 113 & $77.4 \%$ \\
\hline \multirow{2}{*}{$\begin{array}{l}\text { Visual symptoms (any } \\
\text { one) }\end{array}$} & Yes & 80 & $54.8 \%$ \\
\hline & No & 66 & $45.2 \%$ \\
\hline \multirow{2}{*}{ Burning sensations } & Yes & 34 & $23.3 \%$ \\
\hline & No & 112 & $76.7 \%$ \\
\hline \multirow{2}{*}{ Redness } & Yes & 34 & $23.3 \%$ \\
\hline & No & 112 & $76.7 \%$ \\
\hline \multirow{2}{*}{ Head ache } & Yes & 66 & $45.2 \%$ \\
\hline & No & 80 & $54.8 \%$ \\
\hline \multirow{2}{*}{ Eye fatigue } & Yes & 52 & $35.6 \%$ \\
\hline & No & 94 & $64.4 \%$ \\
\hline \multirow{2}{*}{ Double vision } & Yes & 34 & $23.3 \%$ \\
\hline & No & 112 & $76.7 \%$ \\
\hline \multirow{2}{*}{ Focusing problem } & Yes & 51 & $34.9 \%$ \\
\hline & No & 95 & $65.1 \%$ \\
\hline \multirow{2}{*}{ Insomnia } & Yes & 83 & $56.9 \%$ \\
\hline & No & 63 & $43.1 \%$ \\
\hline
\end{tabular}

The association between laptop, smart phone and other gadgets usage and prevalence of MSDs are presented in Table 7. The participants who did not use external mouse while using laptop were more likely to develop MSDs of neck $(\mathrm{p}=0.001)$, shoulder $(p=0.02)$, wrist $/$ hand $(p=0.003)$, upper back $(p=0.009)$, lower back $(p=0.001)$ and Knee $(p=0.01)$. Smart phone use was associated with MSDs of ankle/feet and occurred most commonly among the participants reported using smart phones less than $3 \mathrm{~h}$ per day.

The association between selected factors and the prevalence of visual symptoms and insomnia are summarized in Table 8. Age was associated with prevalence of visual symptoms $(p=0.015)$ and occurred most commonly in 22-24 age group. The 
participants using smart phones more than $6 \mathrm{~h}$ per day were more likely to develop insomnia $(\mathrm{p}=0.007)$.

Table 5. Association between personal characteristics and prevalence of MSDs.

\begin{tabular}{|c|c|c|c|c|c|c|c|c|c|c|c|}
\hline \multirow{2}{*}{ Characteristics } & & \multicolumn{2}{|l|}{ Gender } & \multicolumn{4}{|c|}{ Age (Years) } & \multicolumn{4}{|l|}{ BMI } \\
\hline & & Male & Female & $18-20$ & $20-22$ & $22-24$ & more than 24 & Under weight & Normal weight & Over weight & Obese \\
\hline & No & 22 & 45 & 4 & 24 & 39 & 0 & 11 & 40 & 10 & 6 \\
\hline \multirow[t]{3}{*}{ Neck } & $\%$ & $35.50 \%$ & $53.60 \%$ & $50.00 \%$ & $43.60 \%$ & $48.80 \%$ & $0.00 \%$ & $45.80 \%$ & $44.90 \%$ & $50.00 \%$ & $46.20 \%$ \\
\hline & $P$ & $0.03^{*}$ & & 0.396 & & & & 0.983 & & & \\
\hline & No & 9 & 22 & 1 & 12 & 18 & 0 & 4 & 18 & 7 & 2 \\
\hline \multirow[t]{3}{*}{ Shoulder } & $\%$ & $14.50 \%$ & $26.20 \%$ & $12.50 \%$ & $21.80 \%$ & $22.50 \%$ & $0.00 \%$ & $16.70 \%$ & $20.20 \%$ & $35.00 \%$ & $15.40 \%$ \\
\hline & $P$ & 0.088 & & 0.738 & & & & 0.41 & & & \\
\hline & No & 4 & 12 & 0 & 5 & 11 & 0 & 0 & 12 & 3 & 1 \\
\hline \multirow[t]{3}{*}{ Elbow } & $\%$ & $6.50 \%$ & $14.30 \%$ & $0.00 \%$ & $9.10 \%$ & $13.80 \%$ & $0.00 \%$ & $0.00 \%$ & $13.50 \%$ & $15.00 \%$ & $7.70 \%$ \\
\hline & $P$ & 0.134 & & 0.534 & & & & 0.26 & & & \\
\hline & No & 11 & 19 & 2 & 10 & 18 & 0 & 2 & 20 & 6 & 2 \\
\hline \multirow[t]{3}{*}{ Wrist/ Hand } & $\%$ & $17.70 \%$ & $22.60 \%$ & $25.00 \%$ & $18.20 \%$ & $22.50 \%$ & $0.00 \%$ & $8.30 \%$ & $22.50 \%$ & $30.00 \%$ & $15.40 \%$ \\
\hline & $P$ & 0.471 & & 0.741 & & & & 0.296 & & & \\
\hline & No & 9 & 34 & 2 & 8 & 32 & 1 & 8 & 29 & 5 & 1 \\
\hline \multirow[t]{3}{*}{ Upper back } & $\%$ & $14.50 \%$ & $40.50 \%$ & $25.00 \%$ & $14.50 \%$ & $40.00 \%$ & $33.30 \%$ & $33.30 \%$ & $32.60 \%$ & $25.00 \%$ & $7.70 \%$ \\
\hline & $P$ & $0.001^{*}$ & & $0.016^{*}$ & & & & 0.29 & & & \\
\hline & No & 9 & 30 & 2 & 14 & 23 & 0 & 6 & 23 & 5 & 5 \\
\hline \multirow[t]{3}{*}{ Lower back } & $\%$ & $14.50 \%$ & $35.70 \%$ & $25.00 \%$ & $25.50 \%$ & $28.80 \%$ & $0.00 \%$ & $25.00 \%$ & $25.80 \%$ & $25.00 \%$ & $38.50 \%$ \\
\hline & $P$ & $0.004^{*}$ & & 0.724 & & & & 0.79 & & & \\
\hline & No & 5 & 8 & 0 & 4 & 9 & 0 & 1 & 9 & 2 & 1 \\
\hline \multirow[t]{3}{*}{ Hip } & $\%$ & $8.10 \%$ & $9.50 \%$ & $0.00 \%$ & $7.30 \%$ & $11.30 \%$ & $0.00 \%$ & $4.20 \%$ & $10.10 \%$ & $10.00 \%$ & $7.70 \%$ \\
\hline & $P$ & 0.76 & & 0.615 & & & & 0.83 & & & \\
\hline & No & 12 & 18 & 3 & 6 & 21 & 0 & 2 & 17 & 9 & 2 \\
\hline \multirow[t]{3}{*}{ Knee } & $\%$ & $19.40 \%$ & $21.40 \%$ & $37.50 \%$ & $10.90 \%$ & $26.30 \%$ & $0.00 \%$ & $8.30 \%$ & $19.10 \%$ & $45.00 \%$ & $15.40 \%$ \\
\hline & $P$ & 0.75 & & 0.075 & & & & $0.02^{*}$ & & & \\
\hline & No & 4 & 17 & 0 & 7 & 13 & 1 & 2 & 14 & 4 & 1 \\
\hline \multirow[t]{2}{*}{ Ankle/feet } & $\%$ & $6.50 \%$ & $20.20 \%$ & $0.00 \%$ & $12.70 \%$ & $16.30 \%$ & $33.30 \%$ & $8.30 \%$ & $15.70 \%$ & $20.00 \%$ & $7.70 \%$ \\
\hline & $P$ & $0.01^{*}$ & & 0.463 & & & & 0.61 & & & \\
\hline
\end{tabular}

No: Number of Participants; \%: Percentage of Participants; P: P value of Chi-square test; *: P<0.05

\section{Discussion}

The current study is the first in Saudi Arabia is conducted with an aim to assess the prevalence of computer-related health problems among University students. Majority of the participants in our study reported using desktop less than $3 \mathrm{~h}$ and laptop for 3-6 $\mathrm{h}$ daily. The computer usage of our participants is comparable with that of bank employees in Saudi Arabia (Mean=8.2 h per day) and information technology professionals in India (Mean=7.7 $\mathrm{h}$ per day) [24,25]. The prevalence of MSDs in any one body region among students in this study was $52.7 \%$. The prevalence rate reported in our study is lesser than the prevalence reported by university students in Malaysia (88\%) [26]. A possible 
explanation for the decreased rate reported in our study may be due to use of a case definition to screen MSD.

Table 6. Association between desk top usage and prevalence of MSDs.

\begin{tabular}{|c|c|c|c|c|c|c|c|c|c|c|c|c|c|c|c|c|}
\hline & & \multicolumn{3}{|c|}{ Computer Use (Years) } & $\begin{array}{l}\text { Daily D } \\
\text { (Hours) }\end{array}$ & Desk Tc & op Use & $\begin{array}{l}\text { Daily } \\
\text { (Hours) }\end{array}$ & \multicolumn{2}{|c|}{ Key board } & \multicolumn{3}{|c|}{ Daily Mouse use (Hours) } & \multicolumn{3}{|c|}{ Take Breaks } \\
\hline \multicolumn{2}{|c|}{ Characteristics } & $\begin{array}{l}\text { Less } \\
\text { than } 5 \\
\text { years }\end{array}$ & $\begin{array}{l}\text { From } \\
5-10 \\
\text { years }\end{array}$ & $\begin{array}{l}\text { From } \\
10-15 \\
\text { years }\end{array}$ & $\begin{array}{l}\text { Less } \\
\text { than } 3\end{array}$ & $\begin{array}{l}\text { From } \\
3-6\end{array}$ & $\begin{array}{l}\text { More } \\
\text { than } 6\end{array}$ & $\begin{array}{l}\text { Less } \\
\text { than } 3\end{array}$ & $\begin{array}{l}\text { From } \\
3-6\end{array}$ & $\begin{array}{l}\text { More } \\
\text { than } 6\end{array}$ & $\begin{array}{l}\text { Less } \\
\text { than } 3\end{array}$ & $\begin{array}{l}\text { From } \\
3-6\end{array}$ & $\begin{array}{l}\text { More } \\
\text { than } 6\end{array}$ & $\begin{array}{l}\text { Once in } \\
2 \mathrm{hr}\end{array}$ & $\begin{array}{l}\text { Once in } \\
2-4 \mathrm{hr}\end{array}$ & $\begin{array}{l}\text { Once in } \\
4 \mathrm{hr} \text { or } \\
\text { more }\end{array}$ \\
\hline \multirow{3}{*}{ Neck } & No & 17 & 26 & 24 & 61 & 4 & 2 & 61 & 4 & 2 & 63 & 4 & 0 & 36 & 24 & 7 \\
\hline & $\%$ & $63.00 \%$ & $38.80 \%$ & $46.20 \%$ & $48.00 \%$ & $28.60 \%$ & $40.00 \%$ & $48.00 \%$ & $28.60 \%$ & $40.00 \%$ & $49.20 \%$ & $25.00 \%$ & $0.00 \%$ & $48.00 \%$ & $42.10 \%$ & $50.00 \%$ \\
\hline & $\mathrm{P}$ & 0.104 & & & 0.369 & & & 0.369 & & & 0.079 & & & 0.756 & & \\
\hline \multirow{3}{*}{ Shoulder } & No & 9 & 9 & 13 & 30 & 0 & 1 & 30 & 0 & 1 & 30 & 1 & 0 & 20 & 5 & 6 \\
\hline & $\%$ & $33.30 \%$ & $13.40 \%$ & $25.00 \%$ & $23.60 \%$ & $0.00 \%$ & $20.00 \%$ & $23.60 \%$ & $0.00 \%$ & $20.00 \%$ & $23.40 \%$ & $6.30 \%$ & $0.00 \%$ & $26.70 \%$ & $8.80 \%$ & $42.90 \%$ \\
\hline & $\mathrm{P}$ & 0.073 & & & 0.122 & & & 0.122 & & & 0.217 & & & $0.005^{*}$ & & \\
\hline \multirow{3}{*}{ Elbow } & No & 5 & 4 & 7 & 14 & 1 & 1 & 14 & 1 & 1 & 14 & 2 & 0 & 10 & 2 & 4 \\
\hline & $\%$ & $18.50 \%$ & $6.00 \%$ & $13.50 \%$ & $11.00 \%$ & $7.10 \%$ & $20.00 \%$ & $11.00 \%$ & $7.10 \%$ & $20.00 \%$ & $10.90 \%$ & $12.50 \%$ & $0.00 \%$ & $13.30 \%$ & $3.50 \%$ & $28.60 \%$ \\
\hline & $P$ & 0.163 & & & 0.73 & & & 0.73 & & & 0.86 & & & $0.017^{*}$ & & \\
\hline \multirow{3}{*}{$\begin{array}{l}\text { Hand } \\
\text { Wrist }\end{array}$} & No & 8 & 10 & 12 & 28 & 1 & 1 & 28 & 1 & 1 & 28 & 2 & 0 & 23 & 3 & 4 \\
\hline & $\%$ & $29.60 \%$ & $14.90 \%$ & $23.10 \%$ & $22.00 \%$ & $7.10 \%$ & $20.00 \%$ & $22.00 \%$ & $7.10 \%$ & $20.00 \%$ & $21.90 \%$ & $12.50 \%$ & $0.00 \%$ & $30.70 \%$ & $5.30 \%$ & $28.60 \%$ \\
\hline & $P$ & 0.239 & & & 0.42 & & & 0.42 & & & 0.525 & & & $0.001^{*}$ & & \\
\hline \multirow{3}{*}{$\begin{array}{l}\text { Upper } \\
\text { back }\end{array}$} & No & 12 & 10 & 21 & 39 & 3 & 1 & 39 & 3 & 1 & 40 & 3 & 0 & 22 & 16 & 5 \\
\hline & $\%$ & $44.40 \%$ & $14.90 \%$ & $40.40 \%$ & $30.70 \%$ & $21.40 \%$ & $20.00 \%$ & $30.70 \%$ & $21.40 \%$ & $20.00 \%$ & $31.30 \%$ & $18.80 \%$ & $0.00 \%$ & $29.30 \%$ & $28.10 \%$ & $35.70 \%$ \\
\hline & $P$ & $0.002^{*}$ & & & 0.689 & & & 0.689 & & & 0.384 & & & 0.853 & & \\
\hline \multirow{3}{*}{$\begin{array}{l}\text { Lower } \\
\text { back }\end{array}$} & No & 10 & 13 & 16 & 37 & 1 & 1 & 37 & 1 & 1 & 37 & 2 & 0 & 20 & 13 & 6 \\
\hline & $\%$ & $37.00 \%$ & $19.40 \%$ & $30.80 \%$ & $29.10 \%$ & $7.10 \%$ & $20.00 \%$ & $29.10 \%$ & $7.10 \%$ & $20.00 \%$ & $28.90 \%$ & $12.50 \%$ & $0.00 \%$ & $26.70 \%$ & $22.80 \%$ & $42.90 \%$ \\
\hline & $P$ & 0.154 & & & 0.198 & & & 0.198 & & & 0.26 & & & 0.315 & & \\
\hline \multirow{3}{*}{ Hip/Thigh } & No & 6 & 4 & 3 & 11 & 1 & 1 & 11 & 1 & 1 & 11 & 2 & 0 & 9 & 2 & 2 \\
\hline & $\%$ & $22.20 \%$ & $6.00 \%$ & $5.80 \%$ & $8.70 \%$ & $7.10 \%$ & $20.00 \%$ & $8.70 \%$ & $7.10 \%$ & $20.00 \%$ & $8.60 \%$ & $12.50 \%$ & $0.00 \%$ & $12.00 \%$ & $3.50 \%$ & $14.30 \%$ \\
\hline & $P$ & $0.027^{*}$ & & & 0.66 & & & 0.66 & & & 0.79 & & & 0.18 & & \\
\hline \multirow{3}{*}{ Knee } & No & 13 & 5 & 12 & 25 & 4 & 1 & 25 & 4 & 1 & 27 & 3 & 0 & 19 & 8 & 3 \\
\hline & $\%$ & $48.10 \%$ & $7.50 \%$ & $23.10 \%$ & $19.70 \%$ & $28.60 \%$ & $20.00 \%$ & $19.70 \%$ & $28.60 \%$ & $20.00 \%$ & $21.10 \%$ & $18.80 \%$ & $0.00 \%$ & $25.30 \%$ & $14.00 \%$ & $21.40 \%$ \\
\hline & $P$ & $0.001^{*}$ & & & 0.7 & & & 0.7 & & & 0.75 & & & 0.281 & & \\
\hline \multirow{3}{*}{ Ankle/feet } & No & 7 & 3 & 11 & 19 & 1 & 1 & 19 & 1 & 1 & 19 & 2 & 0 & 12 & 6 & 3 \\
\hline & $\%$ & $25.90 \%$ & $4.50 \%$ & $21.20 \%$ & $15.00 \%$ & $7.10 \%$ & $20.00 \%$ & $15.00 \%$ & $7.10 \%$ & $20.00 \%$ & $14.80 \%$ & $12.50 \%$ & $0.00 \%$ & $16.00 \%$ & $10.50 \%$ & $21.40 \%$ \\
\hline & $P$ & $0.006^{*}$ & & & 0.684 & & & 0.684 & & & 0.817 & & & 0.494 & & \\
\hline
\end{tabular}

No: Number of Participants; \%: Percentage of Participants; P: P value of Chi-square test; ${ }^{*} P<0.05$

In epidemiological studies, it is crucial for the investigator to use a case definition of the ailment to distinguish the healthy from the unhealthy people [27]. Case definitions are the important elements of public health surveillance systems [28]. The World Health Organization (WHO) has emboldened the use of case definitions to facilitate the comparison of surveillance data around the globe [29,30]. This is the first study in Saudi Arabia to use a case definition to assess the prevalence of MSDs. NIOSH case definition for MSD is used 
in this study [21]. This case definition distinguishes MSDs

affecting work from minor complaints.

Table 7. Association between laptop, smart phone and other gadgets usage and prevalence of MSDs.

\begin{tabular}{|c|c|c|c|c|c|c|c|c|c|c|c|c|c|c|c|c|c|}
\hline \multirow{2}{*}{\multicolumn{2}{|c|}{ Characteristics }} & \multirow{2}{*}{$\begin{array}{l}\begin{array}{l}\text { Daily } \\
\text { (Hours) }\end{array} \\
\text { Less } \\
\text { than } 3\end{array}$} & \multirow{2}{*}{$\begin{array}{l}\text { Laptop } \\
\text { From } 3 \\
-6\end{array}$} & \multirow{2}{*}{$\begin{array}{l}\text { Use } \\
\text { More } \\
\text { than } 6\end{array}$} & \multirow{2}{*}{$\begin{array}{l}\text { Daily } \\
\text { (Hours }\end{array}$} & \multirow{2}{*}{$\begin{array}{l}\text { Key boar } \\
\begin{array}{l}\text { From } 3 \\
-6\end{array}\end{array}$} & \multirow{2}{*}{$\begin{array}{l}\text { d use } \\
\text { More } \\
\text { than } 6\end{array}$} & \multicolumn{2}{|c|}{$\begin{array}{l}\text { Use external } \\
\text { key board }\end{array}$} & \multirow{2}{*}{$\begin{array}{l}\begin{array}{l}\text { Use } \\
\text { Mouse }\end{array} \\
\text { Yes }\end{array}$} & \multirow{2}{*}{$\begin{array}{l}\text { external } \\
\text { No }\end{array}$} & \multicolumn{3}{|c|}{$\begin{array}{l}\text { Daily Smart phone use } \\
\text { (Hours) }\end{array}$} & \multicolumn{3}{|c|}{$\begin{array}{l}\text { Daily I Pad or other } \\
\text { Gadget use (Hours) }\end{array}$} \\
\hline & & & & & & & & Yes & No & & & $\begin{array}{l}\text { Less } \\
\text { than } 3\end{array}$ & $\begin{array}{l}\text { From } 3 \\
-6\end{array}$ & $\begin{array}{l}\text { More } \\
\text { than } 6\end{array}$ & $\begin{array}{l}\text { Less } \\
\text { than } 3\end{array}$ & $\begin{array}{l}\text { From } 3 \\
-6\end{array}$ & $\begin{array}{l}\text { More } \\
\text { than } 6\end{array}$ \\
\hline \multirow{3}{*}{ Neck } & No & 25 & 24 & 18 & 37 & 22 & 8 & 13 & 54 & 14 & 53 & 5 & 24 & 38 & 60 & 6 & 1 \\
\hline & $\%$ & $\begin{array}{l}56.80 \\
\%\end{array}$ & $\begin{array}{l}37.50 \\
\%\end{array}$ & $\begin{array}{l}47.40 \\
\%\end{array}$ & $\begin{array}{l}42.50 \\
\%\end{array}$ & $\begin{array}{l}48.90 \\
\%\end{array}$ & $\begin{array}{l}57.10 \\
\%\end{array}$ & $\begin{array}{l}33.30 \\
\%\end{array}$ & $\begin{array}{l}50.50 \\
\%\end{array}$ & $\begin{array}{l}25.90 \\
\%\end{array}$ & $\begin{array}{l}57.60 \\
\%\end{array}$ & $\begin{array}{l}33.30 \\
\%\end{array}$ & $\begin{array}{l}51.10 \\
\%\end{array}$ & $\begin{array}{l}45.20 \\
\%\end{array}$ & $\begin{array}{l}46.90 \\
\%\end{array}$ & $\begin{array}{l}40.00 \\
\%\end{array}$ & $\begin{array}{l}33.30 \\
\%\end{array}$ \\
\hline & $\mathrm{P}$ & 0.138 & & & 0.529 & & & 0.06 & & 0.001 * & & 0.479 & & & 0.798 & & \\
\hline \multirow{3}{*}{ Shoulder } & No & 8 & 16 & 7 & 14 & 14 & 3 & 7 & 24 & 6 & 25 & 4 & 9 & 18 & 27 & 4 & 0 \\
\hline & $\%$ & $\begin{array}{l}18.20 \\
\%\end{array}$ & $\begin{array}{l}25.00 \\
\%\end{array}$ & $\begin{array}{l}18.40 \\
\%\end{array}$ & $\begin{array}{l}16.10 \\
\%\end{array}$ & $\begin{array}{l}31.10 \\
\%\end{array}$ & $\begin{array}{l}21.40 \\
\%\end{array}$ & $\begin{array}{l}17.90 \\
\%\end{array}$ & $\begin{array}{l}22.40 \\
\%\end{array}$ & $\begin{array}{l}11.10 \\
\%\end{array}$ & $\begin{array}{l}27.20 \\
\%\end{array}$ & $\begin{array}{l}26.70 \\
\%\end{array}$ & $\begin{array}{l}19.10 \\
\%\end{array}$ & $\begin{array}{l}21.40 \\
\%\end{array}$ & $\begin{array}{l}21.10 \\
\%\end{array}$ & $\begin{array}{l}26.70 \\
\%\end{array}$ & $0.00 \%$ \\
\hline & $P$ & 0.616 & & & 0.135 & & & 0.55 & & $0.02 *$ & & 0.8 & & & 0.58 & & \\
\hline \multirow{3}{*}{ Elbow } & No & 5 & 6 & 5 & 8 & 6 & 2 & 3 & 13 & 5 & 11 & 3 & 6 & 7 & 14 & 2 & 0 \\
\hline & $\%$ & $\begin{array}{l}11.40 \\
\%\end{array}$ & $9.40 \%$ & $\begin{array}{l}13.20 \\
\%\end{array}$ & $9.20 \%$ & $\begin{array}{l}13.30 \\
\%\end{array}$ & $\begin{array}{l}14.30 \\
\%\end{array}$ & $7.70 \%$ & $\begin{array}{l}12.10 \\
\%\end{array}$ & $9.30 \%$ & $\begin{array}{l}12.00 \\
\%\end{array}$ & $\begin{array}{l}20.00 \\
\%\end{array}$ & $\begin{array}{l}12.80 \\
\%\end{array}$ & $8.30 \%$ & $\begin{array}{l}10.90 \\
\%\end{array}$ & $\begin{array}{l}13.30 \\
\%\end{array}$ & $0.00 \%$ \\
\hline & $P$ & 0.835 & & & 0.706 & & & 0.44 & & 0.614 & & 0.367 & & & 0.796 & & \\
\hline \multirow{3}{*}{$\begin{array}{l}\text { Hand } \\
\text { Wrist }\end{array}$} & No & 8 & 15 & 7 & 14 & 14 & 2 & 7 & 23 & 4 & 26 & 3 & 7 & 20 & 28 & 2 & 0 \\
\hline & $\%$ & $\begin{array}{l}18.20 \\
\%\end{array}$ & $\begin{array}{l}23.40 \\
\%\end{array}$ & $\begin{array}{l}18.40 \\
\%\end{array}$ & $\begin{array}{l}16.10 \\
\%\end{array}$ & $\begin{array}{l}31.10 \\
\%\end{array}$ & $\begin{array}{l}14.30 \\
\%\end{array}$ & $\begin{array}{l}17.90 \\
\%\end{array}$ & $\begin{array}{l}21.50 \\
\%\end{array}$ & $7.40 \%$ & $\begin{array}{l}28.30 \\
\%\end{array}$ & $\begin{array}{l}20.00 \\
\%\end{array}$ & $\begin{array}{l}14.90 \\
\%\end{array}$ & $\begin{array}{l}23.80 \\
\%\end{array}$ & $\begin{array}{l}21.90 \\
\%\end{array}$ & $\begin{array}{l}13.30 \\
\%\end{array}$ & $0.00 \%$ \\
\hline & $\mathrm{P}$ & 0.744 & & & 0.107 & & & 0.63 & & 0.003 * & & 0.479 & & & 0.5 & & \\
\hline \multirow{3}{*}{$\begin{array}{l}\text { Upper } \\
\text { back }\end{array}$} & No & 13 & 19 & 11 & 22 & 17 & 4 & 11 & 32 & 9 & 34 & 6 & 12 & 25 & 39 & 3 & 1 \\
\hline & $\%$ & $\begin{array}{l}29.50 \\
\%\end{array}$ & $\begin{array}{l}29.70 \\
\%\end{array}$ & $\begin{array}{l}28.90 \\
\%\end{array}$ & $\begin{array}{l}25.30 \\
\%\end{array}$ & $\begin{array}{l}37.80 \\
\%\end{array}$ & $\begin{array}{l}28.60 \\
\%\end{array}$ & $\begin{array}{l}28.20 \\
\%\end{array}$ & $\begin{array}{l}29.90 \\
\%\end{array}$ & $\begin{array}{l}16.70 \\
\%\end{array}$ & $\begin{array}{l}37.00 \\
\%\end{array}$ & $\begin{array}{l}40.00 \\
\%\end{array}$ & $\begin{array}{l}25.50 \\
\%\end{array}$ & $\begin{array}{l}29.80 \\
\%\end{array}$ & $\begin{array}{l}30.50 \\
\%\end{array}$ & $\begin{array}{l}20.00 \\
\%\end{array}$ & $\begin{array}{l}33.30 \\
\%\end{array}$ \\
\hline & $P$ & 0.9 & & & 0.327 & & & 0.84 & & 0.009 * & & 0.561 & & & 0.694 & & \\
\hline \multirow{3}{*}{$\begin{array}{l}\text { Lower } \\
\text { back }\end{array}$} & No & 13 & 13 & 13 & 19 & 14 & 6 & 8 & 31 & 5 & 34 & 4 & 14 & 21 & 36 & 3 & 0 \\
\hline & $\%$ & $\begin{array}{l}29.50 \\
\%\end{array}$ & $\begin{array}{l}20.30 \\
\%\end{array}$ & $\begin{array}{l}34.20 \\
\%\end{array}$ & $\begin{array}{l}21.80 \\
\%\end{array}$ & $\begin{array}{l}31.10 \\
\%\end{array}$ & $\begin{array}{l}42.90 \\
\%\end{array}$ & $\begin{array}{l}20.50 \\
\%\end{array}$ & $\begin{array}{l}29.00 \\
\%\end{array}$ & $9.30 \%$ & $\begin{array}{l}37.00 \\
\%\end{array}$ & $\begin{array}{l}26.70 \\
\%\end{array}$ & $\begin{array}{l}29.80 \\
\%\end{array}$ & $\begin{array}{l}25.00 \\
\%\end{array}$ & $\begin{array}{l}28.10 \\
\%\end{array}$ & $\begin{array}{l}20.00 \\
\%\end{array}$ & $0.00 \%$ \\
\hline & $\mathrm{P}$ & 0.27 & & & 0.186 & & & 0.307 & & 0.001 * & & 0.838 & & & 0.456 & & \\
\hline \multirow{3}{*}{ Hip/Thigh } & No & 2 & 9 & 2 & 7 & 4 & 2 & 4 & 9 & 3 & 10 & 3 & 5 & 5 & 12 & 1 & 0 \\
\hline & $\%$ & $4.50 \%$ & $\begin{array}{l}14.10 \\
\%\end{array}$ & $5.30 \%$ & $8.00 \%$ & $8.90 \%$ & $\begin{array}{l}14.30 \\
\%\end{array}$ & $\begin{array}{l}10.30 \\
\%\end{array}$ & $8.40 \%$ & $5.60 \%$ & $\begin{array}{l}10.90 \\
\%\end{array}$ & $\begin{array}{l}20.00 \\
\%\end{array}$ & $\begin{array}{l}10.60 \\
\%\end{array}$ & $6.00 \%$ & $9.40 \%$ & $6.70 \%$ & $0.00 \%$ \\
\hline & $P$ & 0.153 & & & 0.749 & & & 0.709 & & 0.276 & & 0.138 & & & 0.81 & & \\
\hline \multirow{3}{*}{ Knee } & No & 10 & 14 & 6 & 17 & 10 & 3 & 7 & 23 & 5 & 25 & 3 & 8 & 19 & 27 & 2 & 1 \\
\hline & $\%$ & $\begin{array}{l}22.70 \\
\%\end{array}$ & $\begin{array}{l}21.90 \\
\%\end{array}$ & $\begin{array}{l}15.80 \\
\%\end{array}$ & $\begin{array}{l}19.50 \\
\%\end{array}$ & $\begin{array}{l}22.20 \\
\%\end{array}$ & $\begin{array}{l}21.40 \\
\%\end{array}$ & $\begin{array}{l}17.90 \\
\%\end{array}$ & $\begin{array}{l}21.50 \\
\%\end{array}$ & $9.30 \%$ & $\begin{array}{l}27.20 \\
\%\end{array}$ & $\begin{array}{l}20.00 \\
\%\end{array}$ & $\begin{array}{l}17.00 \\
\%\end{array}$ & $\begin{array}{l}22.60 \\
\%\end{array}$ & $\begin{array}{l}21.10 \\
\%\end{array}$ & $\begin{array}{l}13.30 \\
\%\end{array}$ & $\begin{array}{l}33.30 \\
\%\end{array}$ \\
\hline & $\mathrm{P}$ & 0.696 & & & 0.933 & & & 0.639 & & $0.01 *$ & & 0.748 & & & 0.67 & & \\
\hline \multirow{3}{*}{ Ankle/feet } & No & 6 & 11 & 4 & 11 & 10 & 0 & 8 & 13 & 5 & 16 & 6 & 4 & 11 & 20 & 1 & 0 \\
\hline & $\%$ & $\begin{array}{l}13.60 \\
\%\end{array}$ & $\begin{array}{l}17.20 \\
\%\end{array}$ & $\begin{array}{l}10.50 \\
\%\end{array}$ & $\begin{array}{l}12.60 \\
\%\end{array}$ & $\begin{array}{l}22.20 \\
\%\end{array}$ & $0.00 \%$ & $\begin{array}{l}20.50 \\
\%\end{array}$ & $\begin{array}{l}12.10 \\
\%\end{array}$ & $9.30 \%$ & $\begin{array}{l}17.40 \\
\%\end{array}$ & $\begin{array}{l}40.00 \\
\%\end{array}$ & $8.50 \%$ & $\begin{array}{l}13.10 \\
\%\end{array}$ & $\begin{array}{l}15.60 \\
\%\end{array}$ & $6.70 \%$ & $0.00 \%$ \\
\hline & $\mathrm{P}$ & 0.642 & & & 0.09 & & & 0.203 & & 0.16 & & 0.009 & & & 0.5 & & \\
\hline
\end{tabular}

No: Number of Participants; \%: Percentage of Participants; P: P value of Chi-square test; *: $P<0.05$ 
The neck compliant was the most prevalent MSDs $(45.9 \%)$ reported by our participants. Our finding is supported by the study of Lorusso et al. among Italian university students, who also observed neck pain as the most prevalent symptom (69\%) but is slightly higher than the rate reported in our study [17].
Korhonen et al. in their study among office employees reported that neck pain was associated with ergonomically deficient computer workstation setup and individual factors like gender and smoking.

Table 8. Association between selected factors and prevalence of visual symptoms and insomnia.

\begin{tabular}{|c|c|c|c|c|c|c|c|}
\hline \multicolumn{2}{|c|}{ Characteristics } & \multicolumn{3}{|c|}{ Visual Symptoms } & \multicolumn{3}{|c|}{ Insomnia } \\
\hline & & No & $\%$ & $\mathrm{P}$ & No & $\%$ & $P$ \\
\hline \multirow[t]{2}{*}{ Gender } & Male & 29 & $46.8 \%$ & 0.094 & 31 & $50.0 \%$ & 0.151 \\
\hline & Female & 51 & $60.7 \%$ & & 52 & $61.9 \%$ & \\
\hline \multirow[t]{4}{*}{ Age (Years) } & $18-20$ & 5 & $62.5 \%$ & $0.015^{*}$ & 7 & $87.5 \%$ & 0.252 \\
\hline & $20-22$ & 39 & $70.9 \%$ & & 32 & $58.2 \%$ & \\
\hline & $22-24$ & 35 & $43.8 \%$ & & 43 & $53.8 \%$ & \\
\hline & more than 24 & 1 & $33.3 \%$ & & 1 & $33.3 \%$ & \\
\hline \multirow[t]{4}{*}{ BMI } & Under weight & 18 & $75.0 \%$ & 0.132 & 15 & $62.5 \%$ & 0.38 \\
\hline & Normal weight & 45 & $50.6 \%$ & & 47 & $52.8 \%$ & \\
\hline & Over weight & 9 & $45.0 \%$ & & 11 & $55.0 \%$ & \\
\hline & Obese & 8 & $61.5 \%$ & & 10 & $76.9 \%$ & \\
\hline \multirow[t]{3}{*}{ Computer Use } & Less than 5 years & 17 & $63.0 \%$ & 0.42 & 12 & $44.4 \%$ & 0.11 \\
\hline & From 5 - 10 years & 33 & $49.3 \%$ & & 36 & $53.7 \%$ & \\
\hline & From $10-15$ years & 30 & $57.7 \%$ & & 38 & $73.1 \%$ & \\
\hline \multirow[t]{3}{*}{ Daily Desk Top Use (Hours) } & Less than 3 & 73 & $57.5 \%$ & 0.164 & 75 & $59.1 \%$ & 0.24 \\
\hline & From $3-6$ & 6 & $42.9 \%$ & & 5 & $35.7 \%$ & \\
\hline & More than 6 & 1 & $20.0 \%$ & & 3 & $60.0 \%$ & \\
\hline \multirow[t]{3}{*}{ Daily Laptop Use (Hours) } & Less than 3 & 27 & $61.4 \%$ & 0.306 & 24 & $54.5 \%$ & 0.8 \\
\hline & From $3-6$ & 36 & $56.3 \%$ & & 36 & $56.3 \%$ & \\
\hline & More than 6 & 17 & $44.7 \%$ & & 23 & $60.5 \%$ & \\
\hline \multirow[t]{3}{*}{ Take Breaks } & Once in $2 \mathrm{hr}$ & 40 & $53.3 \%$ & 0.749 & 45 & $60.0 \%$ & 0.69 \\
\hline & Once in $2-4 \mathrm{hr}$ & 31 & $54.4 \%$ & & 30 & $52.6 \%$ & \\
\hline & Once in $4 \mathrm{hr}$ or more & 9 & $64.3 \%$ & & 8 & $57.1 \%$ & \\
\hline \multirow[t]{3}{*}{ Daily Smart phone use (Hours) } & Less than 3 & 8 & $53.3 \%$ & 0.725 & 4 & $26.7 \%$ & $0.007^{*}$ \\
\hline & From $3-6$ & 28 & $59.6 \%$ & & 23 & $48.9 \%$ & \\
\hline & More than 6 & 44 & $52.4 \%$ & & 56 & $66.7 \%$ & \\
\hline \multirow[t]{3}{*}{ Daily I Pad or other Gadget use (Hours) } & Less than 3 & 73 & $57.0 \%$ & 0.11 & 71 & $55.5 \%$ & 0.66 \\
\hline & From $3-6$ & 7 & $46.7 \%$ & & 10 & $66.7 \%$ & \\
\hline & More than 6 & 0 & $0.0 \%$ & & 2 & $66.7 \%$ & \\
\hline
\end{tabular}

No: Number of Participants; \%: Percentage of Participants; P: P value of Chi-square test; *: P<0.05

In our study, female gender was associated with MSDs of neck, upper back, lower back, and ankle/feet regions. In epidemiological studies among college students, Katz et al. described that female students experienced higher occurrence of musculoskeletal problems than male students $[31,32]$. The gender differences could be explained based on psychological and biological mechanism. Females pay more focus on their symptoms and respond seriously than males [33]. A review by 
Tittiranonda et al. explains possible biological differences in females like metabolism, physical structures, and hormonal variations, affecting transmission, sensitivity, and perception of pain [34].

In our study, over weight (high BMI) was associated with Knee MSDs. High BMI increase the force across the weight bearing joints (spine and lower extremities) leading to early degenerative changes [35]. Inadequate rest breaks was associated with MSDs of shoulder, elbow and wrist/hand. Earlier researchers also reported the increased risk of MSDs among the computer users who have constrained rest break opportunities [34,36]. Rest breaks relieves computer user from most of the strain levied by continuous computer work such as muscle fatigue, static posture and decreased blood circulation [37]. With regard to the duration and frequency of rest breaks, researchers recommend short breaks at regular intervals to reestablish the ability of the users to resume their work $[38,39]$.

The participants who did not use external mouse while using laptop were more likely to develop MSDs of upper limb and spine. The fixed nature of monitor and keyboard of the laptop does not allow the user to adjust the relative position between keyboard and monitor for optimum angle, height and distance based on ergonomic recommendations. Hence, the user assume an awkward posture while using laptop that in turn increases the risk of MSDs compared to the desktop use [40]. Use of external keyboard and mouse reduces the static and awkward postures and thereby reduces the risk of MSDs among laptop users [41].

More than half of the participants in our study $(54.8 \%)$ experienced any one visual symptom and the headache being the most common $(45.2 \%)$. This finding is similar to the study by Shantakumari et al. among university students in United Arab Emirates. The visual symptoms were associated with improper viewing distance, presence of glare and inadequate rest breaks during computer use [42]. Proper lighting and use of antiglare screens can eliminate the unwanted glare and reflections on the computer monitor [43]. OSHA recommends viewing distance of 20-40 inches from eyes to front of the computer screen. While gazing at the computer screen the users blink less often would give rise to dryness and fatigue in the eyes. Periodical rest to the eyes, concentrating an object at a farthest distance and blinking often can cut the risk of visual symptoms [44].

The prevalence of sleep disorders among the participants of our study was $56.8 \%$. The prevalence rate reported in our study is lesser than the prevalence reported among Japanese (27\%) and Greek adolescent students (11.4\%) [45,46]. Sleep plays a vital role in physical and mental wellbeing. Sleep improves attention, learning, problem-solving skills and creativity. Inadequate sleep is associated with depression, impulsivity, suicide and low academic performance [47-49]. In our study, insomnia was associated with extensive smart phone use (more than $6 \mathrm{~h}$ per day). The bright light from the electronic devices disturb the circadian rhythm resulting in poor sleep quality. Poor sleep was also a result of mobile phone addiction caused by overuse of mobile phones. Mobile phone overuse increases the risk of mobile phone addiction [45]. The preventive measures of mobile phone addiction should focus on addressing the attitude towards mobile phone usage. The mobile phone users need to understand the importance of sleep and recovery and advice regarding self-imposed limit for smartphone use during night and situations demanding attention [50].

Good understanding regarding ergonomic principles and its timely application are vital to recognize and solve health problems arising from workplace. Hence, it is high time to provide ergonomic training to computer users. Ergonomic orientation and training must be initiated first at the student level to prepare them to get in their selected profession with healthy computer work behaviour [51].

\section{Conclusion}

More than half of the participants reported either musculoskeletal disorders, visual symptoms or sleep disorder. Female gender, laptop use without external mouse and inadequate breaks were associated with MSDs. Extensive smart phone use was associated with sleep disorders. The results of this study highlights some significant issues linked to computer-related health problems among university students. As universities extensively use information and communication technology for teaching, discussion, assessment and evaluation, the safe practice regarding computer use should be addressed in their curriculum at an appropriate level. Moreover, the culture of reporting injuries and relevant issues should be encouraged among the student community. These measures could promote the occupational health of the future work force across the globe.

\section{Acknowledgement}

Finally, the authors would like to thank the Deanship of Scientific Research at Majmaah University for supporting this work under Project Number No. 37/82.

\section{Author Contributions}

All authors contributed to literature review, concept and research design. Dr Sirajudeen, Dr Waly and Mr. Muthusamy performed data collection, data analysis and drafting of manuscript. All the authors reviewed the final manuscript.

\section{References}

1. Sirajudeen MS, Pillai PS. Test-retest reliability of a questionnaire to assess the ergonomic knowledge of computer professionals. Int J Health Rehab Sci 2015; 4: 239-243.

2. Communications and Information Technology Commissions. Computer and internet usage in the Kingdom of Saudi Arabia (2007-2009). Internet Usage in the Kingdom of Saudi Arabia 2009.

3. Stubbs DA. Ergonomics and occupational medicine: future challenges. Occup Med 2000; 50: 277-282. 
4. Sirajudeen MS, Shah UN, Mohan N, Somasekharan P. Content validity of a questionnaire to assess the ergonomic knowledge of computer professionals. Int $\mathrm{J}$ Curr Res Rev 2012; 4: 114-121.

5. Sirajudeen MS, Somasekharan P, Shah UN, Mohan N. Content validity and inter-rater reliability of a checklist to assess the ergonomic practice of computer professionals. Int J Therap Rehab Res 2012; 1: 11-18.

6. Jomoah IM. Work-related health disorders among Saudi computer users. Sci World J 2014.

7. Shrivastava SR, Bobhate PS. Computer related health problems among software professionals in Mumbai: A cross-sectional study. Int J Health Allied Sci 2012; 1: 74-78.

8. Thomée S, Härenstam A, Hagberg M. Computer use and stress, sleep disturbances, and symptoms of depression among young adults-a prospective cohort study. BMC Psychiatry 2012; 12: 176.

9. Sirajudeen MS, Alaidarous M, Waly M, Alqahtani M. Work related musculoskeletal disorders among faculty members of college of applied medical sciences, Majmaah University, Saudi Arabia-a cross-sectional study. Int J Health Sci 2018.

10. Punnett L, Wegman DH. Work-related musculoskeletal disorders: the epidemiologic evidence and the debate. J Electromyogr Kinesiol 2004; 14: 13-23.

11. Da Costa BR, Vieira ER. Risk factors for work-related musculoskeletal disorders: a systematic review of recent longitudinal studies. Am J Ind Med 2010; 53: 285-323.

12. Bernard B, Sauter S, Fine L. Job task and psychosocial risk factors for work related musculoskeletal disorders among newspaper employees. Scand J Work Environ Health 1994; 20: 417-426.

13. Karsh B, Moro FBP, Smith MJ. The efficacy of workplace ergonomic interventions to control musculoskeletal disorders: A critical examination of the peer-reviewed literature. Theoret Issues Ergon Sci 2001; 2: 3-96.

14. Logaraj M, Madhupriya V, Hegde SK. Computer vision syndrome and associated factors among medical and engineering students in Chennai. Ann Med Health Sci Res 2014; 4: 179-185.

15. Labbafinejad Y, Aghilinejad M, Sadeghi Z. Association between duration of daily visual display terminal work and sleep disorders among statistics center staff in Iran. Iran Red Crescent Med J 2010; 12: 419-423.

16. Noack-Cooper KL, Sommerich CM, Mirka GA. College students and computers assessment of usage patterns and musculoskeletal discomfort. Work 2009; 32: 285-298.

17. Lorusso A , Bruno S, Labbate N. Musculoskeletal disorders among university student computer users. Medicina del Lavaro 2009; 100: 29-34.

18. WHO. Obesity: preventing and managing the global epidemic: Report of a WHO consultation. World Health Organ Tech Rep Ser 2000; 894: 253.
19. Kuorinka I, Jonsson B, Kilbom A. Standardized Nordic questionnaires for the analysis of musculoskeletal symptoms. Appl Ergon 1987; 18: 233-237.

20. Trinkoff A, Lipscomb J, Geiger-Brown J, Brady B. Musculoskeletal problems of the neck, shoulder, and back and functional consequences in nurses. Am J Ind Med 2002; 41: 170-178.

21. Bernard B, Sauter S, Fine L, Petersen M, Hales T. Job task and psychosocial risk factors for work-related musculoskeletal disorders among newspaper employees. Scand J Work Environ Health 1994; 20: 417-426.

22. Soldatos CR, Dikeos DG, Paparrigopoulos TJ. The diagnostic validity of the Athens Insomnia Scale. J Psychosom Res 2003; 55: 263-267.

23. Mucsi I, Molnar MZ, Ambrus C, Szeifert L, Kovacs AZ, Zoller R. Restless legs syndrome, insomnia and quality of life in patients on maintenance dialysis. Nephrol Dial Transplant 2005; 20: 571-577.

24. Abul-Qasim J, Hadi S, Al-Saleh M, Abu-Alkhair M, Sutaih M, Karaly A, Al-Tayeb M. Arthralgia in computer users in Almadinah Almunawwarah, Kingdom of Saudi Arabia. J Taibah Univ Med Sci 2014; 9: 250-255.

25. Sirajudeen MS, Pillai PS, Vali GMY. Assessment of knowledge of ergonomics among information technology professionals in India. Int J Health Rehab Sci 2013; 2: 192-197.

26. Rajagopal V, Rosli RM, Rintai P, Rustim N, Benadus R, Usai W. The prevalence of computer-related musculoskeletal pain among college students-a crosssectional study. Am Med J 2012; 3: 33-36.

27. Assuncao AA, Barreto SM, Jardim R. Voice disorder: Case definition and prevalence in teachers. Rev Bras Epidemiol 2007; 10: 625-636.

28. Coggon D. Norms and standards in epidemiology: case definitions. Epidemiol Bull 1999; 20: 12-13.

29. Krause G, Brodhun B, Altmann D, Claus H, Benzler J. Reliability of case definitions for public health surveillance assessed by Round-Robin test methodology. BMC Public Health 2006; 6: 129.

30. Korhonen T, Ketola R, Toivonen R, Luukkonen R, Häkkänen M, Viikari-Juntura E. Work related and individual predictors for incident neck pain among office employees working with video display units. Occup Environ Med 2003; 60: 475-482.

31. Katz JN, Amick BC, Carroll BB, Hollis C, Fossel AH, Coley CM. Prevalence of upper extremity musculoskeletal disorders in college students. Am J Med 2000; 109: 586-588.

32. Chang $\mathrm{CH}$, Amick BC, Menendez CC, Katz JN, Johnson PW, Robertson M. Daily computer usage correlated with undergraduate students' musculoskeletal symptoms. Am J Industrial Med 2007; 50: 481-488.

33. Muller CF. Health care and gender. New York: Russell Sage Foundation 1990. 
34. Tittiranonda P, Burastero S, Rempel D. Risk factors for musculoskeletal disorders among computer users. Occupational Med 1999; 14: 17-38.

35. Viester L, Verhagen EA, Hengel KM, Koppes LL, van der Beek AJ, Bongers PM. The relation between body mass index and musculoskeletal symptoms in the working population. BMC Musculoskeletal Disorders 2013; 14: 238.

36. Bergqvist U, Wolgast E, Nilsson B, Voss $M$. Musculoskeletal disorders among visual display terminal workers: Individual, ergonomic, and work organizational factors. Ergonomics 1995; 38: 763-776.

37. Carter JB, Banister EW. Musculoskeletal problems in VDT work: A review. Ergonomics 1994; 37: 1623-1648.

38. Fisher DL, Andres RO, Airth D, Smith SS. Repetitive motion disorders: The design of optimal rate-rest profiles. Human Factors 1993; 35: 283-304.

39. Henning RA, Jacques P, Kissel GV, Sullivan AB, AlterasWebb SM. Frequent short rest breaks from computer work: Effects on productivity and well-being at two field sites. Ergonomics 1997; 1: 78-91.

40. Harris C, Straker L. Survey of physical ergonomics issues associated with school childrens' use of laptop computers. Int J Industrial Ergonomics 2000; 26: 337-346.

41. Sommerich CM, Starr H, Smith CA, Shivers C. Effects of notebook computer configuration and task on user biomechanics, productivity, and comfort. Int J Industrial Ergonomics 2002; 30: 7-31.

42. Shantakumari N, Eldeeb R, Sreedharan J, Gopal K. Computer use and vision-related problems among university students in Ajman, United Arab Emirate. Ann Med Health Sci Res 2014; 4: 258-263.

43. Loh KY, Reddy SC. Understanding and preventing computer vision syndrome. Malaysian Family Physician 2008; 3: 128-130.

44. Occupational Safety and Health Administration (OSHA). Ergonomics. United States Department of Labor 1997.
45. Tamura H, Nishida T, Tsuji A, Sakakibara H. Association between excessive use of mobile phone and insomnia and depression among Japanese adolescents. Int J Environ Res Public Health 2017; 14: 701.

46. Siomos KE, Braimiotis D, Floros GD, Dafoulis V, Angelopoulos NV. Insomnia symptoms among Greek adolescent students with excessive computer use. Hippokratia 2010; 14: 203-207.

47. National Heart, Lung and Blood Institute. Sleep deprivation and deficiency. U.S. Department of Health \& Human Services 2014.

48. Mindell JA, Owens JA, Carskadon MA. Developmental features of sleep. Child Adolesc Psychiatr Clin N Am 1999; 8: 695-725.

49. Roberts RE, Roberts CR, Chen IG. Impact of insomnia on future functioning of adolescents. J Psychosom Res 2002; 53: 561-569.

50. Thomée S, Härenstam A, Hagberg M. Mobile phone use and stress, sleep disturbances, and symptoms of depression among young adults-a prospective cohort study. BMC Public Health 2011; 11: 66.

51. Sirajudeen MS, Siddik SSM. Knowledge of computer ergonomics among computer science engineering and information technology students in Karnataka, India. Asian J Pharm Res Health Care 2017; 9: 64-70.

\section{*Correspondence to}

Mohamed Sherif Sirajudeen

Department of Physical Therapy and Health Rehabilitation

College of Applied Medical Sciences

Majmaah University

Majmaah, 11952

Saudi Arabia

E-mail:m.sirajudeen@mu.edu.sa 